\title{
EFFECTIVE ELASTIC PROPERTIES OF CRACKED SOLIDS: AN EXPERIMENTAL INVESTIGATION
}

\author{
Céline Mallet, Jérôme Fortin, Yves Guéguen and Frédéric Bouyer \\ Laboratoire de Géologie, Ecole Normale Supérieure, CNRS, UMR 8538, Paris, France \\ mail:mallet@geologie.ens.fr
}

\begin{abstract}
Non Interaction Approximation (NIA) is currently used to relate effective elastic moduli to crack density. It also allows one to identify the anisotropy of the crack network.

We investigated thermally cracked glass sample and quantified the crack network (using SEM). From elastic wave velocity measurements, using EMT, we obtained the crack densities: $\alpha_{I}=0.038$ (horizontal one) and $\alpha_{3}=0.0037$ (vertical one).

From SEM observations and image processing we could identify all cracks, count them and determine independently the crack density. These results agree with the previously determined ones from the elastic wave velocity measurements (within a small uncertainty range).

These results indicate that the NIA is quite accurate in the considered range of crack densities, and elastic wave velocity measurements can provide reliable information on their crack distribution and crack density.
\end{abstract}

\section{Keywords: Damaged materials, Elastic wave velocities, Effective medium model, Crack network.}

1. Introduction. Failure of brittle materials such as glass or rocks results from nucleation and propagation of cracks. Cracks modify also the elastic properties. Kachanov (1980) introduced the crack density tensor, a generalization of the scalar crack density first introduced by Bristow (1960), to quantify the effect of cracks on elastic properties. The scalar crack density is adequate in the isotropic case. For identical penny shaped cracks of radius $a$, its value is $\rho_{c}=N a^{3} / V_{T}$ if $N$ is the number of cracks in the volume $V_{T}$. If cracks have different radii, $\rho_{c}=\Sigma_{i}\left(a_{i}^{3}\right) / V_{T}$.

Experimental results have shown that elastic wave velocities are lower in the presence of cracks (Walsh, 1965). Using Effective Medium Theory and in particular the Non-Interaction Approximation, elastic wave velocities can be interpreted in terms of crack density (Schoenberg, 1980, Sayers and Kachanov, 1995). In the case of an anisotropic medium, the components of the stiffness tensor can be derived from the velocities. Many experimental results on granite (Pratt et al., 1977) or basalt (Vinciguerra et al., 2005, Adelinet et al., 2011, Fortin et al., 2011, Nara et al., 2011) have been interpreted in such a way.

Direct and independent measurements of crack density from Scanning Electron Microscope (SEM) would be welcome to validate the EMT results. Unfortunately, such measurements are not in general available for rocks. This is due to three reasons. First, a reliable estimate of crack size appears to be difficult to obtain. This is due to features such as grain boundaries. Second, the crack network can be modified when the sample is unloaded, in particular if cracks are introduced at high pressure (Fortin et al., 2007). Finally, crack shapes strongly affect the predictions, in addition, the information on them is difficult to obtain, and their quantitative analyses are difficult (although some progress has been made in this direction, (Mear et al., 2007)).

For these reasons, we choose to document crack network and crack density on thermally cracked glass sample. Because of the perfect amorphous structure of initial glass sample, when cracks are introduced, the estimation of their size is possible and the crack shape can be well observed. Also, 
cracks have been introduced at room pressure through thermal shocks that prevents artefacts due to unloading of the sample.

We first determine the crack density from elastic wave measurements in the Non-Interaction Approximation. Then, we use microstructural observations to infer independently the crack density. Finally, we discuss how both results can be compared.

\section{Methodology.}

2.1. Original samples and thermal shock. Original borosilicate glass samples were synthesized by the French Atomic Energy Commission (CEA) laboratory by a reproducible method, under conditions of slow cooling that prevent any crack formation. We used cylinders of $80 \mathrm{~mm}$ length and $40 \mathrm{~mm}$ diameter.

Cracks were introduced in the intact samples by thermal shocking (Ougier-Simonin et al., 2010, Ougier-Simonin et al., 2011). Glass samples were heated in an oven at $1{ }^{\circ} \mathrm{C} / \mathrm{min}$ up to $300^{\circ} \mathrm{C}$, kept at this constant temperature during 2 hours and then, quenched in less than $5 \mathrm{~s}$ into water at room temperature.

It is expected that cracks will nucleate near the sample boundaries due to the high (non-linear) thermal gradient introduced there. However, as discussed below, cracks not only nucleate but propagate. So mostly vertical cracks are expected.

2.2. Elastic wave measurements. Elastic wave velocities of cracked samples have been measured in triaxial cell. 16 ultrasonic piezoelectric sensor were glued directly onto the sample surface. In active mode, a pulse of $250 \mathrm{~V}$ is generated and transmitted to one sensor. The electrical pulse triggers a mechanical vibration that propagates into the medium. The other 15 sensors receive this vibration and transform it into an electrical signal that is amplified at $40 \mathrm{~dB}$. Signals are recorded (Schubnel et al., 2006, Ougier-Simonin et al., 2010), and by measuring the travel time of the elastic pulse through the sample for a known path length, elastic wave velocities are obtained (Birch, 1960, Yin, 1992). We used compressional (P) and shear (S) wave sensors to get the P- and S-wave velocities (Fortin et al., 2006). P sensors were glued at different orientations to get the velocity along the radial axis and the vertical axis. For $\mathrm{S}$ waves, one pair of $\mathrm{S}$ sensor was oriented in a horizontal direction for measuring $\mathrm{S}_{\mathrm{H}}$, and a second pair was oriented in the vertical direction for measuring $\mathrm{S}_{\mathrm{V}}$.

2.3. Microstructural investigation. To observe and document the crack network, four different thin sections were cut from one thermally cracked sample (Figure 1a): a vertical plane section, in the lower half cylinder, and three horizontal plane sections, one at the top of the sample (at $5 \mathrm{~mm}$ from the top), one at $16 \mathrm{~mm}$ from the top, and the last one in the middle of the sample (at $40 \mathrm{~mm}$ from the top).

Crack sizes, crack densities and crack shapes have been investigated on these four sections using a SEM. Images were made with a magnification of $x 60$. Then, the mosaic picture of the entire surface was reconstituted by pasting images end to end. Reconstructed SEM image of the vertical plane is shown on Figure $1 b$.

Two distinct groups of defects are highlighted: bubbles (black dots) and cracks (thin lines). Using image processing, bubbles can be filtered out and the crack network appears more clearly. A few small cracks are missed by the image processing. They can be added from visual direct observations. Then, both length and orientation can be obtained. 
It has to be noticed that even if most cracks are linear, a few of them deviate from a straight line by small angles. We assume that if the angle is less than $10^{\circ}$, a crack is treated as a single one. Figures $1 \mathrm{c}, \mathrm{d}, \mathrm{e}, \mathrm{f}$ show the crack network obtained from image processing on the four investigated sections.

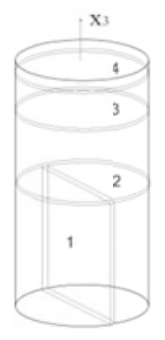

a)

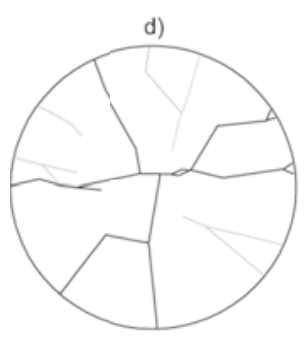

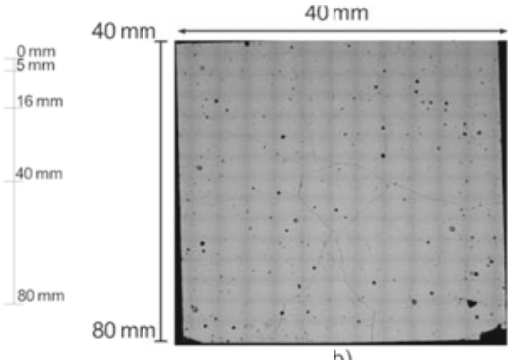

b)

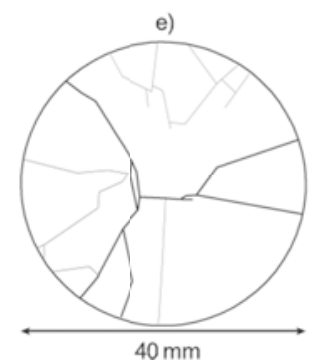

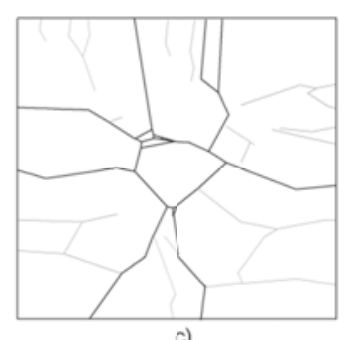

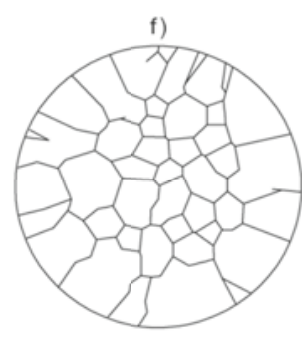

g)

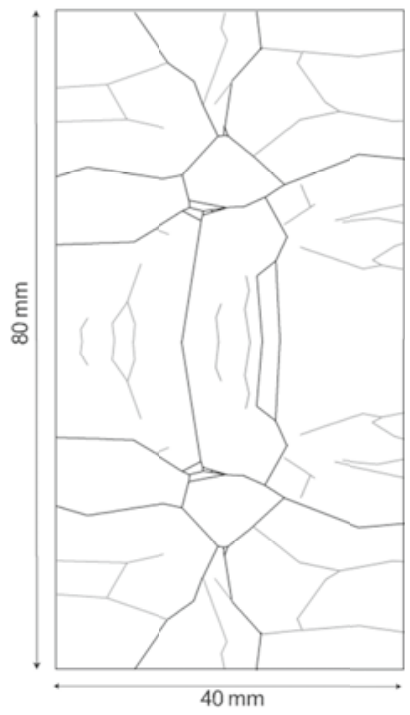

Figure 1. Observation of the crack network. Thin sections are obtained in a thermally cracked sample : (a) position of the vertical section in the lower half cylinder, and position of the three horizontal sections. (b) SEM mosaic in the vertical thin section. (c) image processing of (b). (d) image processing of the horizontal section in the sample center. (e) image processing of the horizontal section at $16 \mathrm{~mm}$ from the top. (f) image processing of the horizontal section at $5 \mathrm{~mm}$ from the sample end. (g) Full sample vertical section reconstructed by symmetry.

\section{Elastic wave velocity data and effective medium model.}

3.1. Elastic wave results. Results are given with an accuracy of $+/-50 \mathrm{~m} / \mathrm{s}$. Velocities are measured at a confining pressure of $10 \mathrm{MPa}$. This pressure value is low so that the pressure effect is weak, but sufficient to get good contacts with transducers. The measured elastic wave velocities are:

$$
\left\{\begin{array}{c}
V_{P_{\text {axial }}}\left(0^{\circ}\right)=6100 \mathrm{~m} / \mathrm{s} \\
V_{P_{\text {radial }}}\left(90^{\circ}\right)=5750 \mathrm{~m} / \mathrm{s} \\
V_{S V}\left(90^{\circ}\right)=2980 \mathrm{~m} / \mathrm{s} \\
V_{S H}\left(90^{\circ}\right)=2900 \mathrm{~m} / \mathrm{s}
\end{array}\right.
$$

3.2. Non-interaction approximation. The effective compliances are derived from the velocities. Assuming a Transversely Isotropic (TI) symmetry (this assumption is checked below), only 5 independent elastic constants have to be considered. If the TI anisotropy is caused by cracks, these 5 constants depend only on 4 parameters (Guéguen and Kachanov, 2011). So only 4 velocity measurements are required: 


$$
\left\{\begin{array}{l}
C_{1111}=\rho V_{11}^{2} \\
C_{3333}=\rho V_{33}^{2} \\
C_{1212}=\rho V_{12}^{2} \\
C_{1313}=\rho V_{13}^{2}
\end{array}\right.
$$

where $\rho$ is the glass density and $V_{i j}$ is the elastic wave velocity along the i-axis and polarized along the j-axis. It means for example that $V_{13}=V_{S V}$, the $\mathrm{S}$ elastic wave, propagating horizontally and vertically polarized (Sayers and Kachanov, 1995).

Compliance tensor is the inverse of the stiffness tensor. Compliance components $S_{i j k l}$ are related to crack density through effective medium theory. In the Non-Interaction Approximation (NIA), from Kachanov (1980) the extra compliances due to cracks is:

where,

$$
\Delta S_{i j k l}=\square\left[\frac{1}{4}\left(\delta_{i k} \alpha_{j l}+\delta_{i l} \alpha_{j k}+\delta_{j k} \alpha_{i l}+\delta_{j l} \alpha_{i k}\right)-\frac{v_{0}}{2} \beta_{i j k l}\right]
$$

$$
h=\frac{32\left(1-v_{0}^{2}\right)}{3\left(2-v_{0}\right) E_{0}}
$$

In this equation, $E_{0}$ and $v_{0}$ represent the Young modulus and the Poisson ratio of the intact glass matrix. $\alpha$ and $\beta$ are the crack density tensors. The second rank crack density tensor is $\alpha=1 / \mathrm{V} \Sigma_{\mathrm{m}}\left(\mathrm{a}^{3} \mathbf{n n}\right)^{(\mathrm{m})}$, and the fourth rank tensor is $\beta=1 / \mathrm{V} \Sigma_{\mathrm{m}}\left(\mathrm{a}^{3} \mathbf{n n n n}\right)^{(\mathrm{m})}$ (where $a^{(m)}$ is the length of the (m) crack and $\boldsymbol{n}$ its normal. The contribution of the beta-term is usually quite small except for the case of fluid-filled cracks where this term needs to be revised and its contribution may, in certain cases, become large (Kachanov, 1993). Retaining $\alpha$ as the sole crack density parameter is an acceptable approximation in dry case (Kachanov, 1980, Kachanov, 1992, Guéguen and Kachanov, 2011).

Tensor $\alpha$ is a second rank tensor, characterized by its three principal values, $\alpha_{1}, \alpha_{2}$ and, $\alpha_{3}$. Note that in this case, the crack density is $\rho_{c}=\alpha_{1}+\alpha_{2}+\alpha_{3}$. The elastic constants depend on 5 parameters: $\mathrm{E}_{0}, \mathrm{v}_{0}, \alpha_{1}, \alpha_{2}$ and, $\alpha_{3}$. In addition, $\alpha_{1=} \alpha_{2}$ so that the number of independent constants is reduced to four. $\alpha_{1}$ corresponds to the vertical crack density and $\alpha_{3}$ is the horizontal crack density.

Then, the overall compliances are given by Guéguen and Kachanov (2011):

$$
\left\{\begin{array}{c}
S_{1111}=\frac{1}{E_{0}}+h \alpha_{1} \\
S_{3333}=\frac{1}{E_{0}}+h \alpha_{3} \\
S_{1212}=\frac{1+v_{0}}{2 E_{0}}+\frac{h}{2} \alpha_{1} \\
S_{1313}=\frac{1+v_{0}}{2 E_{0}}+\frac{h}{4}\left(\alpha_{1}+\alpha_{3}\right)
\end{array}\right.
$$

For our sample, glass density is $2850 \mathrm{Kg} / \mathrm{m}^{3}$. The intact sample Young's modulus and Poisson ratio are obtained from a constant strain rate test on an intact glass sample. They are equal to: $\mathrm{E}_{0}=80 \mathrm{GPa}$, $\mathrm{v}_{0}=0.30$. From the above data, the previous model gives: $\alpha_{1}=0.038, \alpha_{3}=0.0037$, leading to $\rho_{\mathrm{c}}=0.045$ (see Table 1, section 4.3).

Velocities are given with an accuracy of $50 \mathrm{~m} / \mathrm{s}$. We can thus infer that crack densities are known to within $12 \%$. 


\section{Microstructural observations and interpretation.}

4.1. Cracks shape and distribution. Near the cylinder top and bottom ends and around the lateral boundary cracks are expected to nucleate as consequence of thermal shock. An unexpected result is that crack distribution is more homogeneous than the thermal shock would predict (Figures 1c, d, e), if crack nucleation alone was considered. The main boundary effect is observed at the cylinder ends (Figure 1f) where cracks are smaller and more numerous. Note that in this case, the TI symmetry can be applied as the crack network is approximately hexagonal.

The central part of the sample corresponds to Figures 1c, d, e. The crack network has an axial symmetry and as shown below obeys the TI symmetry. This results from the process -quenching- by which cracks are created. For the same reason, the crack network must have the horizontal midcylinder plane as a mirror plane. Based on this assumption, an overall vertical section picture is obtained (Figure 1g). As noted above, if crack nucleation only took place, cracks would be mostly present near the boundaries. This was the expected micro-structure (Ougier-Simonin et al., 2011). However it is clearly not what we observed. The implication is that cracks propagate from the external boundary (where they nucleate) up to the cylinder center.

4.2. Length and orientation: measurements of crack density. From Figure 1, it is possible to get the horizontal crack density $\alpha_{3}$ and the vertical crack density $\alpha_{1}$. Figure $2 \mathrm{a}$ is obtained from the analysis of horizontal sections (Figures 1d, e, f) and shows the evolution of the vertical crack density $\alpha_{1}$ as a function of the azimuthal angle $\varphi$ by angular classes of $45^{\circ}$. As expected, $\alpha_{1}$ does not vary with $\varphi$, the horizontal plane is an "isotropic plane". The overall value of $\alpha_{1}$ obtained from Figures $1 \mathrm{~d}, \mathrm{e}, \mathrm{f}$ is $\alpha_{1}=0.041$ (table 1 ). Horizontal thin sections show the vertical cracks intersections, which are the most important ones.

Let us now examine Figure 1g. Crack radius distribution (Figure 3) is measured on the vertical thin section (Figure 1g) in the entire surface. A few large cracks are found with a radius from $5 \mathrm{~mm}$ to $14 \mathrm{~mm}$. The average radius value is $2.0 \mathrm{~mm}$.
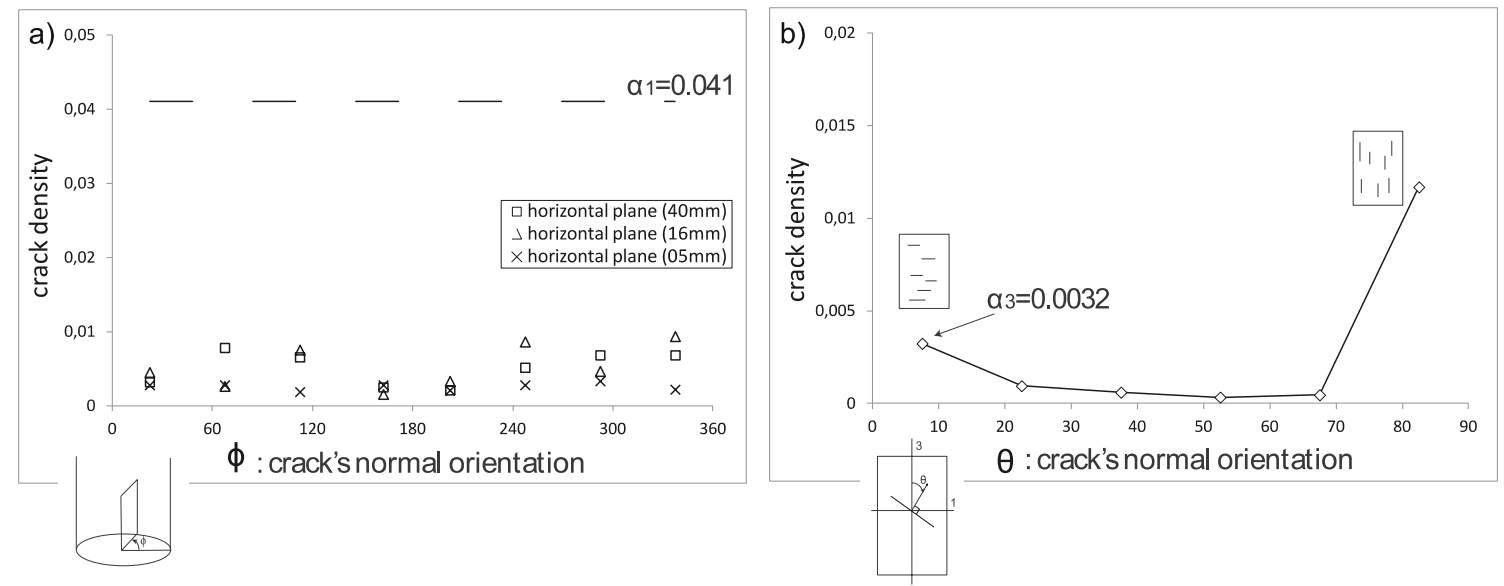

Figure 2. (a) Crack density for Figure 1d,e,f. Cracks are grouped according to their orientation, by angular families of

$45^{\circ}$. Then for each group crack density is calculated. The dashed line at 0.041 represents vertical crack density, $\alpha_{1}$

calculated taking into account all the cracks present on a horizontal section (averaged for the three horizontal sections). (b)

Crack density for Figure 3. Cracks are grouped by angular families of $15^{\circ}$. For each group crack density is calculated. 

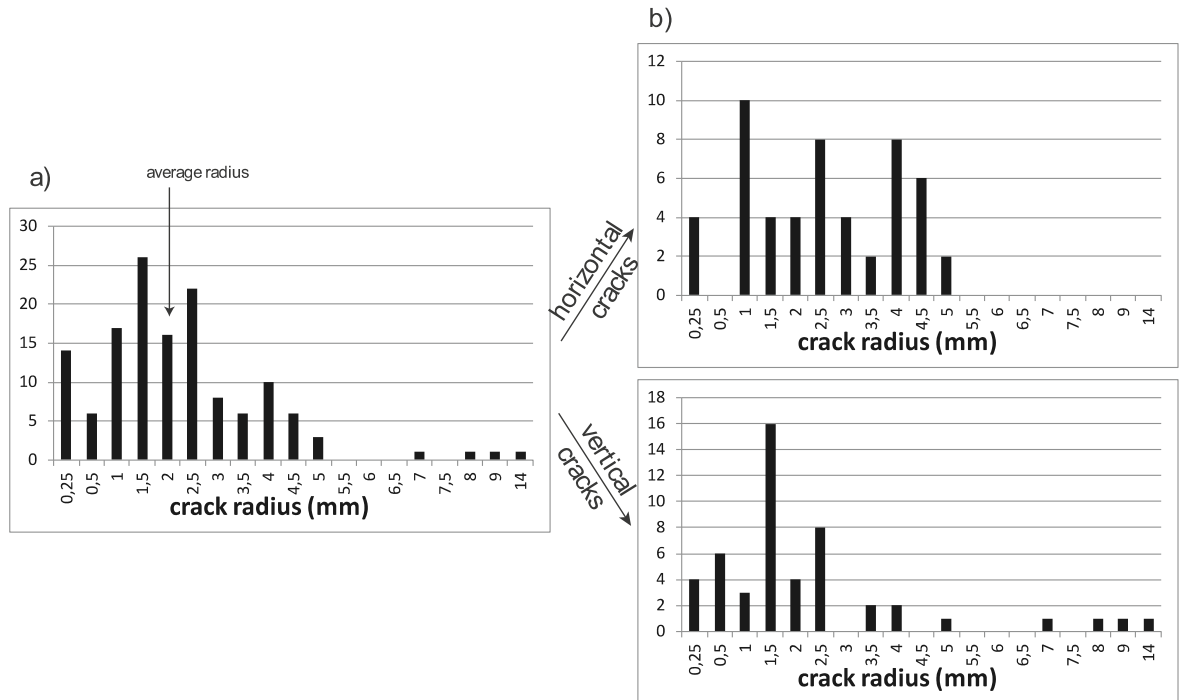

c)

Figure 3. (a) Histogram representing the counting of all the crack radii on the vertical section (Figure 1g). (b) histogram for the vertical and sub-vertical cracks (cracks with a normal oriented between $60-90^{\circ}$ ). (c) Histogram for horizontal and sub-horizontal cracks (cracks with a normal oriented between $0-30^{\circ}$ ).

The distribution is anisotropic and Figure $3 \mathrm{a}$ can be split into two crack families: vertical cracks and horizontal cracks. Both horizontal and vertical families are defined by a $30^{\circ}$ angle range. Note that vertical cracks are longer than horizontal ones (Figures $3 b$ and c). As for horizontal planes, crack density can be obtained for the vertical plane taking into account the crack orientation. This result is shown in Figure 2b. Using Figure 3a, the overall crack density $\rho_{c}$ is found to be 0.017 if the total volume of the cylinder is used. However, considering the volume $\mathrm{V}_{\mathrm{T}}$ of the entire sample is not appropriate since the vertical plane of Figure $1 \mathrm{~g}$ represents only a fraction $f$ of $\mathrm{V}_{\mathrm{T}}$. Therefore, $\rho_{c}=$ $0.017 \mathrm{~m}$ where $m=1 / f$. From Figure $3 \mathrm{c}$, the vertical crack density is $\alpha_{1}=0.012 \mathrm{~m}$. From Figure $3 \mathrm{~b}$, the horizontal crack density is $\alpha_{3}=0.0032$ (indeed all horizontal cracks are seen, thus there is no $\mathrm{m}$ factor). The crack network is clearly characteristic of a transverse isotropic medium. Comparing the overall results of Figures 2a and b, we get $m=3.4$.

4.3. Discussion. Table 1 summarizes crack density results. The accuracy is $12 \%$. Crack densities obtained from microstructural observations and from elastic wave velocity measurements are in very good agreement. These results are however obtained using several theoretical and experimental approximations.

Table 1. Crack densities $\alpha_{1}$ (vertical cracks), $\alpha_{3}$ (horizontal cracks) and global crack density $\rho_{\mathrm{c}}$.

\begin{tabular}{|c|c|c|}
\hline & Thin section measurements & elastic wave velocity inversion \\
\hline$\alpha_{1}$ & 0.041 & $0.038+/-0.004$ \\
$\alpha_{3}$ & 0.0032 & $0.0037+/-0.0004$ \\
$\rho_{\mathrm{c}}$ & 0.047 & $0.045+/-0.005$ \\
\hline
\end{tabular}


NIA Assumptions. Probably the most important assumption is the non-interaction approximation. The results of (Grechka and Kachanov, 2006a, 2006b) show that the NIA remains accurate at crack density lower than 0.14 . In our case, crack densities are always less than 0.14 . This basic assumption is therefore very appropriate and justified.

Another important assumption is that cracks are planar and disc-shaped. Observations made in orthogonal sections validate the important assumption of planar cracks. The disc-shaped assumption cannot be checked. But this assumption is less critical in the sense that equivalent circular cracks are in fact described by EMT (Guéguen and Kachanov, 2011). However, the numerical equivalence is unsolved.

Finally, the transversely isotropic assumption is very well confirmed from the above crack density angular dependence.

Observation of vertical cracks in vertical section. As examined in section 4.2 (Figure 2a), $\alpha_{1}=0.041$ is deduced from Figures $1 \mathrm{~d}$,e,f measurements. This result is in agreement with measurements independently deduced from Figure $1 \mathrm{~g}$ if $m=3.4$. The $m$ factor has been defined as $m=1 / f$, where $f$ is the appropriate fraction of the total volume $\mathrm{V}_{\mathrm{T}}$ to be used in determining crack density from Figure $1 \mathrm{~g}$. Factor $f$ is such that $0<f<1$ so that $m \geq 1$. Although it is difficult to calculate an exact value for $f$, examination of Figures $1 \mathrm{~d}$,e,f symmetry provides some indications. In the TI symmetry ( $\approx$ hexagonal symmetry), the crack network is invariant by a rotation of $2 \pi / 6$ (i.e. $\pi / 3$ ) around the cylinder axis. This suggests that any observation in a vertical plane corresponds to $1 / 3$ of the total crack numbers. From this argument, a value of $m \approx 3$ is expected.

Limits of applicability. We are within the range of applicability of the Non-Interaction Approximation - that constitutes the approximation that is rigorously correct at low crack densities. However, this experimental investigation between microstructure analysis and velocity inversion would be even more valuable if it would be obtained for higher crack densities. Unfortunately, stronger thermal shocks lead to sample failure (Ougier-Simonin et al., 2010). Investigating the upper limit of the model could be done on other materials that support a higher crack density.

\section{Conclusions}

Elastic wave measurements and thin sections measurements have provided independently values of crack density (vertical cracks and horizontal ones) on cracked glass samples. These values agree reasonably well. The crack network is not perfectly homogeneous, but it is approximatively homogeneous. It has a transverse isotropic symmetry as expected. The Non-Interaction Approximation assumption is fulfilled. These results confirm the validity of this approximation and the potential use of elastic wave velocities to infer crack density.

\section{References}

Adelinet, M., C. Dorbath, M. Le Ravalec, J. Fortin, and Y. Guéguen (2011), Deriving microstructure and fluid state within the Icelandic crust from the inversion of tomography data, J. geophys. Res, 38, 3.

Birch, F. (1960), The velocity of compressional waves in rocks to 10 kilobars, part 1, Journal of Applied Mechanics, 65, 1083-1102.

Bristow, J. (1960), Microcracks, and the static and dynamic elastic constants of annealed and heavily cold-worked metals, British Journal of Applied Physics, 11, no 2, p. 81. 
Fortin, J., S. Stanchits, G. Dresen, and Y. Guéguen (2006), Acoustic emission and velocities associated with the formation of compaction bands in sandstone, Journal of Geophysical Research, 111(B10), doi:10.1029/2005JB003,854.

Fortin, J., Y. Guéguen, and A. Schubnel (2007), Effects of pore collapse and grain crushing on ultrasonic velocities and vp/vs, Journal of geophysical research, 112, doi:10.129/2005JB004,005.

Fortin, J., S. Stanchits, S. Vinciguerra, and Y. Guéguen (2011), Influence of thermal and mechanical cracks on permeability and elastic wave velocities in a basalt from mt. etna volcano subjected to elevated pressure, Tectonophysics, 503(1), 60-74.

Grechka, V., and M. Kachanov (2006a), Effective elasticity of rocks with closely spaced and intersecting cracks, Geophysics, 71(3), D85-D91.

Grechka, V., and M. Kachanov (2006b), Effective elasticity of fractured rocks: A snapshot of the work in progress, Geophysics, 71(6), W45-W58.

Guéguen, Y., and M. Kachanov (2011), Effective elastic properties of cracked rocks - an overview, in, mechanics of crustal rocks, CISM Courses and Lectures, Volume 533, 73-125.

Kachanov, M. (1980), Continuum model of medium with cracks, Journal of the engineering mechanics division, 106, 1039-1051.

Kachanov, M. (1992), Effective elastic properties of cracked solids, Appl Mech Rev, 45(8).

Kachanov, M. (1993), Elastic solids with many cracks and related problems, Advances in applied mechanics, 30, $259-445$.

Mear, M. E., I. Sevostianov, and M. Kachanov (2007), Elastic compliances of non-flat cracks, International Journal of Solids and Structures, 44(20), 6412-6427.

Nara, Y., P. G. Meredith, T. Yoneda, and K. Kaneko (2011), Influence of macro-fractures and microfractures on permeability and elastic wave velocities in basalt at elevated pressure, Tectonophysics, 503(1), 52-59.

Ougier-Simonin, A., J. Fortin, Y. Guéguen, A. Schubnel, and F. Bouyer (2010), Cracks in glass under triaxial conditions, International Journal of Engineering Science, 49, 105-121.

Ougier-Simonin, A., Y. Guéguen, J. Fortin, A. Schubnel, and F. Bouyer (2011), Permeability and elastic properties of cracked glass under pressure, Journal of geophysical research, 116, doi:10.1029/2010JB008,077.

Pratt, H., H. Swolfs, W. Brace, A. Black, and J. Handin (1977), Elastic and transport properties of an in situ jointed granite, in International Journal of Rock Mechanics and Mining Sciences \& Geomechanics Abstracts, vol. 14, pp. 35-45, Elsevier.

Sayers, C., and M. Kachanov (1995), Microcrack-induced elastic wave anisotropy of brittle rocks, Journal of Geophysical Research, 100(B3), 4149-4156.

Schoenberg, M. (1980), Elastic wave behavior across linear slip interfaces, The Journal of the Acoustical Society of America, 68, 1516-1521.

Schubnel, A., E. Walker, B. Thompson, J. Fortin, Y. Guéguen, and R. Young (2006), Transient creep, aseismic damage and slow failure in carrara marble 3 deformed across the brittle-ductile transition, Geophysical Research Letters, 33, doi:10.1029/2006GL026,619.

Vinciguerra, S., C. Trovato, P. Meredith, and P. Benson (2005), Relating seismic velocities, thermal cracking and permeability in mt. etna and iceland basalts, International Journal of Rock Mechanics and Mining Sciences, 42(7), 900910 .

Walsh, J. (1965), The effect of cracks on the uniaxial elastic compression of rocks, Journal of Geophysical Research, 70(2), 399-411.

Yin, H. (1992), Acoustic velocity and attenuation of rocks: isotropy, intrinsic anisotropy, and stressinduced anisotropy, Ph.D. thesis. 